\title{
New Symmetry Reductions for some Ordinary Differential Equations
}

\author{
M L GANDARIAS, E MEDINA, C MURIEL \\ Departamento de Matemáticas, Universidad de Cádiz, PO.BOX 40, 11510 Puerto Real, \\ Cádiz, Spain
}

Received April 30, 2001; Revised July 17, 2001; Accepted July 26, 2001

\begin{abstract}
In this work we derive potential symmetries for ordinary differential equations. By using these potential symmetries we find that the order of the ODE can be reduced even if this equation does not admit point symmetries. Moreover, in the case for which the ODE admits a group of point symmetries, we find that the potential symmetries allow us to perform further reductions than its point symmetries. Some diffusion equations admitting an infinite number of potential symmetries and the scaling group as a Lie symmetry are considered and some general results are obtained. For all the equations that we have studied, a set of potential symmetries admitted by the diffusion equation is "inherited" by the ODE that emerges as the reduced equation under the scaling group.
\end{abstract}

\section{Introduction}

It is well known that the knowledge of a one parameter Lie symmetry group of an ordinary differential equation (ODE), allows us to reduce the order of the equation by one. In particular, a first order equation with a known one-parameter symmetry group can be integrated by a single quadrature. For higher dimensional symmetry groups the situation is different. In general it is not possible to reduce the order of an equation invariant under an $r$-parameter symmetry group by $r$ using only quadratures, we need a solvable group. In [2] Bluman and Reid derived an algorithm to find new symmetry groups for ODE's. These new symmetries reduce the order of a given ODE in cases where a direct application of Lie's method fails. These symmetries are in general not equivalent to point symmetries.

In the last few years we have observed a significant progress in the application of symmetries to the study of nonlinear partial differential equations (PDE's) of physical importance, as well as in looking for exact solutions for such equations.

Lie classical symmetries admitted by nonlinear PDE's are useful for finding invariant solutions, as well as to discover whether or not the equation can be linearized by an invertible mapping and to construct an explicit linearization when one exists. Nevertheless an obvious limitation of group-theoretic methods based on local symmetries, is that many PDE's do not have local symmetries. It turns out that PDE's can admit nonlocal symmetries whose infinitesimal generators depend on the integrals of the dependent variables in 
some specific manner. For a given PDE one can find useful nonlocal symmetries by embedding it in an auxiliary "covering" system with auxiliary dependent variables. A point symmetry of the auxiliary system, acting on the space consisting of the independent and dependent variables of the given PDE as well as the auxiliary variables, yields a nonlocal symmetry of the given PDE if it does not project onto a point symmetry acting in its space of the independent and dependent variables.

In [3] there have been obtained two hierarchies of PDE's which are linearizable by two corresponding hierarchies of nonlocal transformations. The first of them is

$$
u_{t}=R^{m}[u]\left(u^{-2} u_{x}\right)_{x}
$$

where

$$
R[u]=D_{x}^{2} u^{-1} D_{x}^{-1} .
$$

Thus, the first three equations in the hierarchy (1.1) take the form

$$
\begin{gathered}
u_{t}=\left(u^{-2} u_{x}\right)_{x} \\
u_{t}=\left(u^{-3} u_{x}\right)_{x x} \\
u_{t}=\left(u^{-4} u_{x x}-3 u^{-5} u_{x}\right)_{x x} .
\end{gathered}
$$

The equations (1.3) are invariant under the classical scaling group and admit an infinite set of potential symmetries which allow us to linearize them. Under the scaling group the sets of equations (1.3) are reduced to a set of ODE's. These ODE's only admit a oneparameter Lie group of point symmetries but "inherit" potential symmetries that allow us to linearize the ODE's, or to reduce the order by two.

The aim of this paper is to study a new class of symmetries for ODE's. Some of these ODE's arise by means of invariance of diffusion equations under the scaling group and, "inherit" a set of potential symmetries. These potentials symmetries lead to further order reductions than the classical Lie symmetries of these equations.

We consider:

- The ODE's arising from $u_{t}=\left(f(u) u_{x}\right)_{x x}$, (this equation is a generalization of the second equation of (1.3)), (Section 2).

- The ODE's arising from the third equation of (1.3), (Section 3).

- A second order ODE which does not admit Lie symmetries, however by using potential symmetries can be reduced to a quadrature, (Section 4).

\section{The diffusion equation $u_{t}=\left(f(u) u_{x}\right)_{x x}$}

We consider the third order diffusion equation

$$
u_{t}=\left(f(u) u_{x}\right)_{x x} .
$$

If $f(u)$ is an arbitrary function, the equation (2.1) admits a three-parameter Lie group with infinitesimal generators

$$
X_{1}=\partial_{x}, \quad X_{2}=\partial_{t}, \quad X_{3}=x \partial_{x}+3 t \partial_{t} .
$$


On the other hand, PDE (2.1) is written in a conserved form. Its associated auxiliary system [1] is given by

$$
\begin{aligned}
& v_{x}=u, \\
& v_{t}=\left(f(u) u_{x}\right)_{x} .
\end{aligned}
$$

We have that equation (2.1) admits a potential symmetry, corresponding to the auxiliary system (2.3), if and only if the function $f(u)$ adopts the form

$$
f(u)=\frac{1}{\left(u^{2}+b u+c\right)^{3 / 2}} \exp \left[k \int \frac{d u}{u^{2}+b u+c}\right] .
$$

The corresponding infinitesimal generators of potential symmetries are listed below. Two cases arise:

Case I- $c, b, k$ arbitrary constants such that $9 b^{2}-36 c-4 k^{2} \neq 0$. The equation (2.1) admits the potential symmetry

$$
X_{s}=v \partial_{x}+\left(k-\frac{3 b}{2}\right) t \partial_{t}-\left(u^{2}+b u+c\right) \partial_{u}-(c x+b v) \partial_{v}
$$

Case II- $b, c, k$ arbitrary constants such that $9 b^{2}-36 c-4 k^{2}=0$. It can be easily seen that in this case we can take without loss of generality

$$
f(u)=\frac{1}{u^{3}}
$$

(by replacing $u$ by $k_{1}\left(u+k_{2}\right)$ being $k_{1}$ and $k_{2}$ certain constants). The equation (2.1) admits the potential symmetry

$$
X_{s}=\eta(t, v) \partial_{x}-\eta_{v}(t, v) u^{2} \partial_{u}
$$

where $\eta(t, v)$ is any particular solution of the linear equation

$$
\eta_{t}+\eta_{v v v}=0
$$

Coming back to the Lie symmetries of (2.1), it is known that an important class of solutions of this equation arises from its invariance under the scaling group $X_{3}(2.2)$, using this invariance we have

$$
u=u(z) \quad z=x t^{-1 / 3}
$$

where $u$ satisfies the ODE

$$
z u_{z}+3\left(f(u) u_{z}\right)_{z z}=0 .
$$

For applications it is important to reduce the order of (2.8). We can show that (2.8) admits a one-parameter Lie group of point transformations if and only if

$$
f(u)=\lambda(u+k)^{\nu}
$$


or the limit case

$$
f(u)=\lambda e^{\nu u},
$$

where $\lambda, k, \nu$ are arbitrary constants. The corresponding generators are

$$
V=z \partial_{z}+\frac{3}{\nu} u \partial_{u}
$$

and

$$
V=z \partial_{z}+\frac{3}{\nu} \partial_{u}
$$

These are the only functional forms of $f(u)$ for which the order of (2.8) can be reduced by Lie's method. However, we next show that it is still possible to reduce the order by using potential symmetries of ODE's. In particular we find:

- A functional form of $f$ for which (2.8) does not admit classical Lie symmetries but the order of (2.8) can be reduced by using potential symmetry.

- A functional form of $f$ for which (2.8) just admits a one-parameter Lie group of symmetries and the reduced equation does not admit Lie symmetries, however using potential symmetries the order of (2.8) can be reduced by two.

These two cases correspond to the previous Cases I and II for which the diffusion equation (2.1) admits potential symmetries.

We start by setting $u=v_{z}$, then (2.8) becomes

$$
\left(3\left(f\left(v_{z}\right) v_{z z}\right)_{z}+z v_{z}-v\right)_{z}=0
$$

we also consider the associated system

$$
\begin{aligned}
& v_{z}-u=0 \\
& 3\left(f(u) u_{z}\right)_{z}+z u-v=0,
\end{aligned}
$$

and the corresponding integrated equation

$$
3\left(f\left(v_{z}\right) v_{z z}\right)_{z}+z v_{z}-v=0 .
$$

As it is indicated above two interesting cases arise:

1.-For

$$
f(u)=\frac{1}{\left(u^{2}+1\right)^{3 / 2}} \exp (k \arctan u),
$$

system (2.12) admits a group of transformations with infinitesimal generator

$$
V=\xi(x, u, v) \partial_{x}+\phi(x, u, v) \partial_{u}+\psi(x, u, v) \partial_{v}
$$

where

$$
\xi=v-\frac{k z}{3}, \quad \phi=-\left(u^{2}+1\right), \quad \psi=-z-\frac{k v}{3} .
$$


This symmetry group is a new symmetry group for (2.8) due to the fact that $\xi$ depends explicitly on $v$. This new group also allows us to reduce the order of (2.8). For example, if $k=0$ the canonical coordinates corresponding to (2.16) :

$$
r=\left(z^{2}+v^{2}\right), \quad w=\arctan \left(\frac{v}{z}\right)
$$

lead to expressing ODE

$$
3\left(\frac{v_{z z}}{\left(v_{z}^{2}+1\right)^{3 / 2}}\right)_{z}+z v_{z}-v=0
$$

in the form

$$
-3 r\left(r^{2} y^{2}+1\right) y_{r r}+9 r^{3} y\left(y_{r}\right)^{2}+9 r^{2} y^{2} y_{r}-9 y_{r}-r^{2} y\left(r^{2} y^{2}+1\right)^{5 / 2}+3 r^{3} y^{5}+12 r y^{3}=0
$$

where $y=w_{r}$.

2.- If we consider $f(u)=u^{-3} \mathrm{ODE}$ (2.8) only admits a one-parameter Lie group of point transformations with infinitesimal generator

$$
V=z \partial_{z}-u \partial_{u}
$$

that allows us to reduce (2.8) to the second order ODE

$$
-y^{6} h^{5}+y^{5} h^{4}-3 y^{2} h h^{\prime \prime}+9 y^{2}\left(h^{\prime}\right)^{2}-9 y^{2} h^{2} h^{\prime}+6 y^{2} h^{4}+27 y h h^{\prime}-27 y h^{3}+36 h^{2}=0,
$$

where $y=x u, w=\log x$ and $w_{y}=h$. This equation (2.21) does not admit any classical Lie symmetry. Nevertheless (2.8) admits a set of "potential" symmetries derived by considering the associated system (2.12). System (2.12) admits a new group of symmetries as in (2.15) with infinitesimals

$$
\xi=k_{1} z+\eta(v), \quad \phi=-k_{1} u-\eta_{v}(v) u^{2}, \quad \psi=0
$$

where $\eta(v)$ satisfies

$$
\eta_{v} v+3 \eta_{v v v}-\eta=0 .
$$

A solution for (2.23) is $\eta=k_{2} v$. Consequently the integrated equation (2.13) admits a two-parameter Lie group of point transformations with infinitesimals

$$
V_{1}=z \partial_{z}, \quad V_{2}=v \partial_{z}
$$

As the Lie-bracket is $\left[V_{1}, V_{2}\right]=-V_{2}$ we can reduce (2.13) first by $V_{2}$ and then by $V_{1}$. The canonical coordinates for $V_{2}$ are $w=\frac{z}{v}, y=v$, thus, (2.13) is reduced to the second order Bessel ODE

$$
3 y h^{\prime \prime}+9 h^{\prime}+y^{2} h=0,
$$


where $h=w_{y}$ and 'stands by $\frac{d}{d y}$. Then, (2.25) inherits the Lie symmetry $V_{1}$, whose projection is $\hat{V}_{1}=h \partial_{h}$ (it is clear that invariance under $\hat{V}_{1}$ is just a consequence from being (2.25) a linear equation). This symmetry allows us to reduce (2.25) to the first order ODE,

being $t=y, g=\frac{h^{\prime}}{h}$.

$$
3 t g^{\prime}+3 t g^{2}+9 g+t^{2}=0
$$

\section{Remark}

By making in (2.8) $f(u)=u^{-3}, u=v_{z}$, it can be written in the conserved form

$$
\left[3\left(\left(v_{z}\right)^{-3} v_{z z}\right)_{z}+z v_{z}-v\right]_{z}=0 .
$$

The integrated equation with $c=0$ has the form (2.13), with $f\left(v_{z}\right)=v_{z}^{-3}$. We have found that equation (2.13) admits two generators $V_{1}$ and $V_{2}$. On the other hand, it can be easily seen that the equation (2.26) admits the generator $V_{1}$. Besides, the process for the order reduction from (2.26) to (2.8) has been done by using the Lie symmetry $\partial_{v}$ of equation (2.26). Since $\left[\partial_{v}, V_{1}\right]=0, V_{1}$ is inherited as a Lie symmetry of equation (2.8). The corresponding inherited symmetry, $z \partial_{z}-u \partial_{u}$, is the only Lie symmetry admitted by (2.8).

The same process can not be done for the other admitted Lie symmetry of (2.13) because the vector field $V_{2}=v \partial_{z}$ is not a Lie symmetry for equation (2.26). In fact, it is a conditional symmetry for the equation (2.26) because it transforms only a class of solutions of (2.26) into solutions of (2.26), that is those solutions that verify equation (2.13).

\section{The fourth order diffusion equation $u_{t}=\left[\left(u^{-2}\right)_{x x} u^{-1}\right]_{x x}$}

Another interesting equation, from the point of view of the symmetry reductions is the fourth order diffusion equation

$$
u_{t}=\left[\left(u^{-2}\right)_{x x} u^{-1}\right]_{x x},
$$

related to the third member of the hierarchy (1.1) through the change of variables $t^{\prime}=-2 t$. This equation admits the four parameter group of infinitesimal generators

$$
X_{1}=\partial_{x}, \quad X_{2}=\partial_{t}, \quad X_{3}=x \partial_{x}-u \partial_{u}, \quad X_{4}=4 t \partial_{t}+u \partial_{u}
$$

An important class of solutions of (3.1) arises from its invariance under the scaling group $X=m X_{3}+X_{4}$, i.e.

$$
u=w(z) t^{\frac{1-m}{4}}, \quad z=x t^{-\frac{m}{4}}
$$

where $w$ satisfies the ODE

$$
m\left(z w_{z}+w\right)-w+4\left[\left(w^{-2}\right)_{z z} w^{-1}\right]_{z z}=0 .
$$

We can show that ODE (3.2) only admits a Lie group of point transformations of generator

$$
V=z \partial_{z}-w \partial_{w}
$$


This symmetry allows us to reduce (3.2) to the third order ODE

$$
\begin{aligned}
& -1+m-h^{7} y^{7}+h^{6} y^{2}\left(-24+(-7+m) y^{4}\right)+3 h^{5} y\left(16+(-7+2 m) y^{4}\right) \\
& -40 h^{\prime 2}+120 y^{2} h^{\prime 3}+h^{4}\left(-48+5(-7+3 m) y^{4}+48 y^{2} h^{\prime}\right)-80 y h^{\prime} h^{\prime \prime} \\
& +h^{3} y\left(-176 h^{\prime}+y\left(5(-7+4 m) y+32 h^{\prime \prime}\right)\right)+8 h^{(3)}+h\left(224 y h^{\prime 2}-48 h^{\prime \prime}\right. \\
& \left.-80 y^{2} h^{\prime} h^{\prime \prime}+y\left(-7+6 m+16 h^{(3)}\right)\right)+h^{2}\left(136 h^{\prime}-96 y^{2} h^{\prime 2}\right. \\
& \left.+y\left(-16 h^{\prime \prime}+y\left(-21+15 m+8 h^{(3)}\right)\right)\right)=0,
\end{aligned}
$$

where $y=w z$ and $h=-\frac{d}{d y}(\ln w)$. This equation does not admit any classical Lie symmetry. Nevertheless, (3.2) admits a set of "potential" symmetries derived by considering the associated system

$$
\begin{aligned}
& v_{z}-w=0 \\
& m z w-v+4\left[\left(w^{-2}\right)_{z z} w^{-1}\right]_{z}=0 .
\end{aligned}
$$

In fact, (3.3) has a group of symmetries with infinitesimals

$$
\xi=k_{1} z+\eta(v), \quad \phi=-k_{1} w-\eta_{v}(v) w^{2}, \quad \psi=0,
$$

where $\eta(v)$ satisfies the linear equation

$$
\eta_{v} v-8 \eta_{v v v v}-m \eta=0 .
$$

A particular nontrivial solution of the equation (3.5) can be found if $m=0,1,2, \ldots$ In fact, by making the change of variables

$$
\eta(v)=v^{m}+\phi_{1}(v)
$$

the equation (3.5) becomes

$$
-m \phi_{1}+v \phi_{1 v}+8 m(m-1)(m-2)(m-3) v^{m-4}+8 \phi_{1 v v v v}=0 .
$$

If $m=0,1,2,3, \phi_{1}=0$ is a solution of (3.6), so we have that for (3.5)

$$
\eta=v^{m} \text {. }
$$

If $m \neq 0,1,2,3$, we take

$$
\phi_{1}(v)=c_{1} v^{m-4}+\phi_{2}(v),
$$

where $c_{1}$ is a parameter that we will determine later. By substituting (3.7) into (3.6) we obtain

$$
\begin{aligned}
& -8 c_{1}(m-4)(m-5)(m-6)(m-7) v^{m-8}-4\left[c_{1}+2 m(m-1)(m-2)(m-3)\right] v^{m-4} \\
& -m \phi_{2}+v \phi_{2 v}-8 \phi_{2 v v v v}=0 .
\end{aligned}
$$


Thus, it is clear that if $m=4,5,6,7$, by choosing $c_{1}=-2 m(m-1)(m-2)(m-3)$ and $\phi_{2}=0, \phi_{1}$ is a solution of (3.6), consequently

$$
\eta=v^{m}-2 m(m-1)(m-2)(m-3) v^{m-4}, \quad m=4,5,6,7,
$$

is a solution of (3.5). Proceeding in the same way, we obtain a nontrivial solution of (3.5) for each nonnegative integer $m$. We denote these solutions as $\eta_{m}(v)$. We have then, that for these values of $m$, the integrated equation

$$
m z v_{z}-v+4\left[\left(v_{z}^{-2}\right)_{z z} v_{z}^{-1}\right]_{z}=0
$$

admits the two parameter Lie group of infinitesimals

$$
V_{1}=z \partial_{z}, \quad V_{2}=\eta_{m}(v) \partial_{z}
$$

As $\left[V_{1}, V_{2}\right]=-V_{2}$, we can reduce (3.8) first by $V_{2}$ and then by $V_{1}$. For example, for $m=0,1,2,3$ we reduce (3.8) by $V_{2}$ to the linear third order ODE

$$
\begin{aligned}
& h\left(64 m-96 m^{2}+32 m^{3}-y^{4}\right)+48\left(-m+m^{2}\right) y h^{\prime} \\
& +32 m y^{2} h^{\prime \prime}+8 y^{3} h^{(3)}=0
\end{aligned}
$$

with $y=v, h=\frac{d}{d y}\left(\frac{z}{v^{m}}\right)$, and then (3.9) inherits the symmetry group $\hat{V}_{1}=h \partial_{h}$ that allows us to reduce (3.9) to the second order ODE,

$$
\begin{aligned}
& 64 m-96 m^{2}+32 m^{3}+48 g(-1+m) m t+32 g^{2} m t^{2}+8 g^{3} t^{3} \\
& -t^{4}+32 m t^{2} g^{\prime}+24 t^{3} g g^{\prime}+8 t^{3} g^{\prime \prime}=0
\end{aligned}
$$

with $t=y, g=\frac{h^{\prime}}{h}$. In the same way, for $m=4,5,6,7$ we reduce (3.8) by $V_{2}$ to the linear third order ODE

$$
\begin{aligned}
& h\left(-46080 m+112896 m^{2}-103936 m^{3}+47040 m^{4}-11200 m^{5}+1344 m^{6}\right. \\
& \left.-64 m^{7}+52 m y^{4}-74 m^{2} y^{4}+20 m^{3} y^{4}+2 m^{4} y^{4}-y^{8}\right) \\
& +8 y\left(1440 m h^{\prime}-3288 m^{2} h^{\prime}+2700 m^{3} h^{\prime}-1020 m^{4} h^{\prime}+180 m^{5} h^{\prime}\right. \\
& -12 m^{6} h^{\prime}-6 m y^{4} h^{\prime}+6 m^{2} y^{4} h^{\prime}-192 m y h^{\prime \prime}+400 m^{2} y h^{\prime \prime} \\
& -280 m^{3} y h^{\prime \prime}+80 m^{4} y h^{\prime \prime}-8 m^{5} y h^{\prime \prime}+4 m y^{5} h^{\prime \prime}+12 m y^{2} h^{(3)}-22 m^{2} y^{2} h^{(3)} \\
& \left.+12 m^{3} y^{2} h^{(3)}-2 m^{4} y^{2} h^{(3)}+y^{6} h^{(3)}\right)=0
\end{aligned}
$$


with $y=v, h=\frac{d}{d y}\left(\frac{z}{v^{m}-2 m(m-1)(m-2)(m-3) v^{m-4}}\right)$, and then (3.10) inherits the symmetry group $\hat{V}_{1}=h \partial_{h}$ that allows us to reduce (3.10) to the second order ODE,

$$
\begin{aligned}
& -11200 m^{5}+1344 m^{6}-64 m^{7}-t^{8}-m\left(46080-52 t^{4}\right)+2 m^{4}\left(23520+t^{4}\right) \\
& +4 m^{3}\left(-25984+5 t^{4}\right)-2 m^{2}\left(-56448+37 t^{4}\right)-8 t\left(4 g^{2} m t(48-100 m\right. \\
& \left.+70 m^{2}-20 m^{3}+2 m^{4}-t^{4}\right)-g^{3} t^{2}\left(12 m-22 m^{2}+12 m^{3}-2 m^{4}+t^{4}\right) \\
& +3 g\left(2 ( - 1 + m ) m \left(240-308 m+142 m^{2}-28 m^{3}+2 m^{4}-t^{4}-t^{2}\left(12 m-22 m^{2}\right.\right.\right. \\
& \left.\left.+12 m^{3}-2 m^{4}+t^{4}\right) g^{\prime}\right)-t\left(-4 m\left(48-100 m+70 m^{2}-20 m^{3}+2 m^{4}-t^{4}\right) g^{\prime}\right. \\
& \left.\left.+t\left(12 m-22 m^{2}+12 m^{3}-2 m^{4}+t^{4}\right) g^{\prime \prime}\right)\right)=0
\end{aligned}
$$

with $t=y, g=\frac{h^{\prime}}{h}$.

Summarizing, making use of the potential symmetries of ordinary equations, we have found that we can reduce the problem of looking for solutions of the fourth order diffusion equation, of the form

$$
u(x, t)=t^{\frac{1-m}{4}} w\left(\frac{x}{t^{\frac{m}{4}}}\right) \quad m=0,1,2, \ldots,
$$

to the problem of solving a second order ordinary differential equation and two quadratures.

\section{Integration of equations without Lie symmetries by means of potential symmetries}

We are interested in ODE's which do not admit any Lie symmetry, but by means of potential symmetries can be reduced to quadratures. Let us consider the following second order differential equation:

$$
e^{\frac{1}{u}+x}\left(-u^{4}-u^{5}+2 u^{3} u_{x}-u_{x}^{2}-3 u u_{x}^{2}+u^{2}\left(2 u_{x}+u_{x x}\right)\right)=u^{5}+u^{6}-u^{3} u_{x} .
$$

Equation (4.1) has no Lie symmetries. However, it can be reduced to quadratures in the following way: By means of the transformation $u=v_{x}$ equation (4.1) becomes the third order differential equation:

$$
e^{\frac{1}{v_{x}}+x}\left(-v_{x}^{4}-v_{x}^{5}+2 v_{x}^{3} v_{x x}-v_{x x}^{2}-3 v_{x} v_{x x}^{2}+v_{x}^{2}\left(2 v_{x x}+v_{x x x}\right)\right)=v_{x}^{5}+v_{x}^{6}-v_{x}^{3} v_{x x} .
$$

Equation (4.2) can be expressed in conserved form as follows:

$$
D_{x}\left(\frac{-v_{x}^{2}-v v_{x}^{3}+e^{\frac{1}{v_{x}}+x}\left(-v_{x}^{2}-v_{x}^{3}+v_{x x}\right)-v_{x}^{3} x}{v_{x}^{3}}\right)=0 .
$$


Let us consider any second order equation associated to equation (4.2):

$$
-v_{x}^{2}-v v_{x}^{3}+e^{\frac{1}{v_{x}}+x}\left(-v_{x}^{2}-v_{x}^{3}+v_{x x}\right)-v_{x}^{3} x=C v_{x}^{3}
$$

where $C$ is an arbitrary constant. It can be checked that equation (4.4) admits

$$
X_{1}=e^{-v} \partial_{x} \quad \text { and } \quad X_{2}=-e^{-v}(v+x+1) \partial_{x}+e^{-v} \partial_{v}
$$

as Lie symmetries. Then, $X_{1}$ and $X_{2}$ are potential symmetries of equation (4.1). Since $\left[X_{1}, X_{2}\right]=0$, any of the two Lie symmetries can be used to reduce the order of equation (4.4) and the reduced equation inherits the other symmetry as Lie symmetry. If, for instance, $X_{1}$ is used to reduce the order of equation (4.4), by means of the change of variables $\left\{y=v, \alpha=e^{v} x, w=\alpha_{y}=e^{v}\left(\frac{1}{v_{x}}+x\right)\right\}$, the following reduced equation is obtained:

$$
\left(-1+e^{w e^{-y}}\right) w-e^{w e^{-y}} w_{y}-e^{y}\left(e^{w e^{-y}}+y+C\right)=0 .
$$

The Lie symmetry of equation (4.6) inherited from $X_{2}$ becomes:

$$
\hat{X}_{2}=e^{-y} \partial_{y}+\left(-1+e^{-y} w\right) \partial_{w} .
$$

By means of the canonical coordinates $\left\{z=e^{-y} w+y, \beta=e^{y}\right\}$ for $\hat{X}_{2}$, equation (4.6) can be solved by quadrature:

$$
\beta_{z}=-\frac{e^{z}}{z+C}
$$

Let us denote by $\beta=H\left(z, C_{1}\right)$ its general solution. From $e^{y}=H\left(e^{-y} w+y, C_{1}\right), w$ can be locally expressed as $w=G\left(y, C_{1}\right)$. Since $w=\alpha_{y}$, the general solution of equation (4.4), and hence the solution of equation (4.1), has been obtained through two successive quadratures.

There exists a procedure to reduce the order of equation (4.1) based on the existence of $C^{\infty}$-symmetries. The concept of $C^{\infty}$-symmetry is similar to the concept of Lie symmetry but it is based on a different way of prolonging vector fields. For a function $\lambda \in C^{\infty}\left(M^{(1)}\right)$ we define the $n$-th order $\lambda$-prolongation of a vector field $X=\xi(x, u) \partial_{x}+\eta(x, u) \partial_{u}$, as the following vector field:

$$
X^{[\lambda,(n)]}=\sum_{i=0}^{n}\left(D_{x}+\lambda\right)^{i}\left(\eta(x, u)-\xi(x, u) u_{1}\right) \partial u_{i}+\xi(x, u) D_{x},
$$

where $D_{x}$ denotes the total derivative operator with respect to $x$. The $C^{\infty}\left(M^{(1)}\right)$-symmetries of an equation $\Delta\left(x, u^{(n)}\right)=0$ are the vector fields $X$ for which there exists $\lambda \in C^{\infty}\left(M^{(1)}\right)$ such that $X^{[\lambda,(n)]}\left(\Delta\left(x, u^{(n)}\right)\right)=0$, when $\Delta\left(x, u^{(n)}\right)=0$.

An algorithm that let us reduce the order of the equation can be associated to a given $C^{\infty}$-symmetry. It is based on the construction of invariants of $X^{[\lambda,(n)]}$ by derivation of lower order invariants. This method, as well as equivalent definitions of $C^{\infty}$-symmetries, can be consulted in [6].

We show here how potential symmetries can be recovered as $C^{\infty}$-symmetries of equation (4.1). This is a particular case of the use of the $C^{\infty}$-symmetries method to recover 
lost symmetries arising in equations with non-solvable symmetries algebras. Theoretical results related to the general case can be consulted in [7].

First we observe that equation (4.2) keeps $X_{1}$ and $X_{2}$ as Lie symmetries, and it has an additional Lie symmetry, $X_{3}=\partial_{v}$. The reduced equation obtained by using $X_{3}$ is equation (4.1). It can be checked that the following relations hold:

$$
\begin{aligned}
& {\left[X_{1}, X_{2}\right]=0} \\
& {\left[X_{1}, X_{3}\right]=X_{1}} \\
& {\left[X_{2}, X_{3}\right]=X_{1}+X_{2}}
\end{aligned}
$$

and when $X_{3}$ is used to reduce the order, neither $X_{1}$ nor $X_{2}$ are inheritable symmetries for equation (4.1). In fact, we have showed that equation (4.1) has no Lie symmetries. However, since $\left[X_{1}, X_{3}\right]=X_{1}$, the Lie symmetry $X_{1}$ is inheritable as a $C^{\infty}$-symmetry. The corresponding inherited $C^{\infty}$-symmetry for equation (4.1) is $\hat{X}_{1}=\partial_{x}+u^{2} \partial_{u}$ for the function $\lambda_{1}=-u$.

The $C^{\infty}$-symmetry $\hat{X}_{1}$ can be used to reduce the order of equation (4.1). By means of the change of variables $\left\{y=x+\frac{1}{u}, \beta=x, \mu=\frac{\beta-y}{\beta_{y}}=\frac{u_{x}}{u^{3}}-\frac{1}{u}\right\}$ the corresponding $\hat{X}_{1}$-reduced equation is the following:

$$
-e^{-y}+\mu+\mu e^{-y}-\mu^{2}-\mu \mu_{y}=0 .
$$

The lost symmetry $X_{2}$ can also be recovered as $C^{\infty}$-symmetry of equation (4.8). It takes the form $\hat{X}_{2}=-\partial_{y}+(-1+\mu) \partial_{\mu}$, for the function $\lambda_{2}=\frac{1}{\mu}$, and can be used to solve equation (4.8) by quadrature. In fact, in terms of variables $\left\{s=e^{y}(\mu-1), r=e^{s-y}\right\}$ equation (4.8) becomes

$$
s r_{s}+e^{s}=0 .
$$

When the general solution of equation (4.8) is obtained, through the quadrature (4.9), in the form $\mu=\mathcal{H}(y, C)$, we get the general solution of equation (4.1), in variables $\{y, \beta\}$, by solving the first order linear equation $\beta_{y}=(\beta-y) / \mathcal{H}(y, C)$. In conclusion, equation (4.1) that lacks Lie symmetries, can be solved, by means of $C^{\infty}$-symmetries, by two quadratures.

An algorithmic procedure, based on the use and determination of integrating factors, has been introduced to reduce the order of ODE's [5]. The $C^{\infty}$-symmetries provide a new algorithmic method of reduction. The main advantage of using the reduction based on the existence of $C^{\infty}$-symmetries is that $C^{\infty}$-symmetries can be determined through a welldefined algorithm while conserved forms (4.3) needed to calculate potential symmetries are usually difficult to find.

\section{Conclusions}

In this paper, we have considered a family of diffusion PDE's which admit an infinite number of potential symmetries as well as point symmetries. An important class of solutions of these equations arises from its invariance under the scaling group, using this invariance we get a family of ODE's. Knowing the importance of reducing the order of these ODE's, we have derived potential symmetries for them, as well as for some other interesting ODE's. We have found: 
- ODE's, such as (2.1), where $f$ adopts the form (2.14) for which (2.1) does not admit point symmetries, but whose order can be reduced by using a potential symmetry.

- ODE's, such as (3.2) and (2.1), where $f$ adopts the form (2.6), which just admit a oneparameter Lie group of point symmetries and the corresponding reduced equations do not admit Lie symmetries. Nevertheless we have used potential symmetries to reduce the order by two as well as to linearize them.

- ODE's such as (4.1) which does not admit any Lie symmetry however by means of potential symmetries can be integrated by quadratures.

\section{References}

[1] G.W. Bluman, and S. Kumei, Symmetries and Differential Equations, Springer Berlin (1989).

[2] G.W.Bluman, and , G.R. Reid, IMA J. App. Math., (1988) 40 87-94.

[3] N. Euler, M.L. Gandarias, M. Euler, and O. Lindblom, Auto-Hodograph Transformations for a hierarchy of Nonlinear Evolution Equations, J. Math. Anal. Appl. 257 (2001), 21-28.

[4] M.L. Gandarias, E. Medina, and C. Muriel, Potential Symmetries for Some Ordinary Differential Equations To appear in Nonlinear Analysis.

[5] P.E. Hydon, Symmetry Methods and Differential Equations, Cambridge University Press (2000).

[6] C. Muriel and J.L. Romero, New Methods of Reduction for Ordinary Differential Equations, IMA J. Appl. Math.66 (2001), 111-125.

[7] C. Muriel and J.L. Romero, $C^{\infty}-$ Symmetries and Nonsolvable Symmetry Algebras. To appear in IMA J. Appl. Math. 